\title{
Performance Analysis of Repetition-Coding and Space-Time-Block-Coding as Transmitter Diversity Schemes for Indoor Optical Wireless Communications
}

\author{
Tingting Song, Ampalavanapillai Nirmalathas, Christina Lim, Elaine Wong, Ka-Lun Lee, Yang Hong, \\ Kamal Alameh and Ke Wang
}

\begin{abstract}
The benefits of $2 \times 1$ multiple-inputs-single-output scheme for transmitter diversity in the infrared indoor optical wireless communication link are theoretically investigated. The performance of repetition-coding (RC) and Alamouti-type realvalued space-time-block-coding (STBC) as effective transmitter diversity schemes is systematically compared under conditions of channel gain variation caused by the degradation in the received optical power due to the blocking of one optical beam of the optical wireless channel. It is shown that the linear addition of channel gains in the RC scheme outperforms the root-sum-square of channel gains in the STBC scheme with regards to the bit-errorrate (BER) performance. Proof-of-concept experiments are carried out with both schemes under emulated scenarios of channel blockage. The RC scheme exhibits better BER performance when observed experimentally, validating the proposed theoretical model for the two spatial diversity schemes. To understand the performance of $\mathrm{RC}$ and STBC schemes against the optical delay caused by the two optical channel path difference within one-bit interval, both schemes were experimentally investigated using on-off-keying modulation, and results show that RC still outperforms STBC. Both theoretical and experimental results indicate that $\mathrm{RC}$ has better robustness to channel blockage and differential channel paths induced optical delay.
\end{abstract}

Index Terms - Indoor optical wireless communications, transmitter diversity, repetition-coding, space-time-block-coding

\section{INTRODUCTION}

I $\mathrm{N}$ future scenarios of indoor communications, dramatic increase in both the volume of data traffic and speed of connectivity is widely predicted due to the increasing real-time high-speed information exchanges of end-users as well as emerging ultra-broadband applications, such as virtual reality and augmented reality (VR and AR) [1-3]. Generally, such high-speed access to indoor connectivity will need to be provided wirelessly, as it offers significantly improved flexibility. Compared to the widely-used radio frequency (RF) wireless technologies, such as Wi-Fi and millimeter-wave, the optical wireless communications (OWC) technology is a promising alternative [1]. Optical wireless channel with narrow beams offers inherent physical layer security due to signal confinement within the beam, immunity to electromagnetic interferences due to optical transmission, scalable bandwidth delivered via unregulated optical spectrum and the ability to utilize the existing and new fiber infrastructure of optical access and in-building distribution networks [4-5].

The most popular realization of OWC with high-speed wireless connectivity is based on the line-of-sight (LOS) link configuration. The LOS link has high optical power efficiency and low multipath distortion, yielding a high signal-to-noiseratio (SNR) performance at the receiver, which facilates higher achievable data rates. However, to establish such a LOS link, strict optical alignment is required, and the optical wireless channel is also susceptible to optical beam blockage, arising from objects and mobile users. To provide enhanced robustness for the indoor OWC systems, other link configurations can be used, such as the diffused and the scattered links where the reflected lights from the diffusely reflecting surfaces are utilized [6-7]. However, these links are subjected to multiple path fading, severe optical losses, and significant background light noises due to the wide field-of-view (FOV) receivers that contribute to the performance degradation. Consequently, optical transmission power beyond the laser safety limit and a compromise on achievable data rate is often required [8-10].

To achieve a high data rate indoor OWC link fully compliant with the laser safety regulation, a number of LOS link configurations with robustness against physical optical beam blockages have been proposed. In [11-12], a backup mm-wave $\mathrm{RF}$ system with footprint covering the entire optical beam coverage area has been reported, which can be activated on demand to address the narrow pencil beam blockage. Besides, in [13], multiple spatial light modulators (SLMs) based beam steering base stations with wide FOV to broadcast light have been proposed, which can support beam coverage within entire room and the potential for a backup OWC link for beam blockage. To provide OWC link redundancy, spatial diversity can be introduced in the LOS link configuration to enhance the channel reliability [14-15]. To achieve spatial diversity in the OWC downlink, multiple-inputs-single-output (MISO) and multiple-inputs-multiple-outputs (MIMO) channel configurations are two widely-used options, where the MISO configuration achieves transmitter diversity, and the MIMO configuration achieves the combination of transmitter diversity 
and receiver diversity [15-16]. Compared to the MIMO configuration, the MISO configuration only requires single receiver, which can reduce the complexity and power consumption to achieve compact and portable end-users, and the cost of extra transmitters can be shared by multiple endusers. Therefore, in this paper, we investigate transmitter diversity schemes with the MISO configuration in LOS based infrared indoor OWC systems. When an optical beam is partially blocked at the receiver, i.e., the FOV of receiver is not fully blocked, the induced optical power degradation can be alleviated with the cooperation of optical power from extra transmitters. In such case, if the required optical power is satisfied at the receiver side, a high data rate link can still be maintained. On the other hand, when no optical beam blockage exists, the optical beam coverage area can be broadened by steering the beams to different directions, and hence supporting better user mobility [12-13].

Regarding the signal coding scheme for transmitter diversity in conventional RF wireless systems, both repetition-coding (RC) and Alamouti orthogonal space-time-block-coding (STBC) schemes have been proposed and widely used in MIMO or MISO configurations, where identical signals are transmitted in the RC scheme while orthogonal signals are transmitted in the STBC scheme. It has been shown that the STBC scheme offers the capability of combating channel fading, whilst the RC scheme fails to provide diversity gains due to its potential destructive interference caused by the coherent addition of different RF signal fields [17]. Similarly, in coherent OWC systems, the STBC scheme has been studied in both heterodyne free space optical (FSO) links to combat turbulence-induced fading [18], and homodyne indoor OWC links to increase the system capacity and the coverage [19]. However, in practical indoor OWC scenarios, due to the requirements of low system complexity and low cost, intensity modulation/direct detection (IM/DD) is preferred. Different from RF systems or coherent OWC systems, in IM/DD based indoor OWC systems, optical signal intensities, rather than signal fields, are added at the receiver [17]. In addition, the channel characteristics in indoor OWC systems are highly distinct from outdoor FSO links, where the dominant limiting source in outdoor FSO link is channel fading governed by turbulent conditions [20-22], whilst the optical propagation loss and the background light noise are the key limiting factors in indoor OWC links [23-24]. Furthermore, additional optical propagation loss can be introduced by the optical beam blockage due to the mobility of indoor users. For indoor OWC systems, LED-arrays and lasers are two typical optical sources. In LED-arrays based systems, RC or STBC scheme together with advanced modulation formats and digital signal processing technologies have been carried out to improve the system performance [25-27]. In such transmitter diversity schemes, the spatial dimension has been used to provide redundancy for reliable communication, and often, the data rate is limited [2829] by the the lower modulation bandwidths of current LEDs [30]. Therefore, for higher data rate transmissions, it is necessary to further investigate transmitter diversity techniques, including both STBC and RC schemes, to reduce the impact of optical beam blockage and extend the beam coverage area in indoor infrared based OWC systems, where broader bandwidth lasers are commonly used.

Previously, we have experimentally demonstrated $10 \mathrm{~Gb} / \mathrm{s}$ $2 \times 1$ MISO indoor OWC systems employing RC and STBC schemes, and preliminary results have indicated the tolerance of both schemes to the optical beam blockage [31]. In this paper, we further develop a theoretical framework to investigate the capabilities of the RC and STBC schemes in general infrared indoor MISO OWC systems. The theoretical framework of the bit-error-rate (BER) performance with respect to the transmitter diversity technique, the channel gains, the possible beam blockage, and the received optical power, is established. Verification experiments are carried out with both RC and STBC schemes, in which the optical beam blockage scenarios are emulated by different optical transmission power distributions. The impact of optical delay caused by the optical channel path difference in the RC and STBC schemes is also investigated. Experimental results align with the theoretical study, and show that the RC scheme outperforms the STBC scheme by $\sim 2 \mathrm{~dB}$ in the receiver sensitivity. Besides, the same receiver sensitivity is maintained for different power distributions in the RC scheme due to the same SNR with a fixed total received optical power, while the BER performance varies with the transmitted power distributions in the STBC scheme. Furthermore, experimental results for the impact of channel propagation delay show that within one-bit interval optical delay using the on-off-keying (OOK) modulation, RC always outperforms STBC with better receiver sensitivity. In this paper, our work mainly focuses on the theoretical and experimental analyses of the two coding schemes for indoor downlink with transmitter diversity, and consequently we have not investigated the uplink scenario. The compatible infrared OWC uplink approaches has been reported in $[11,13,32]$.

\section{THEORETICAL MODELS AND SIMULATION RESULTS}

The schematic block diagrams of RC and STBC schemes based $2 \times 1$ MISO infrared indoor OWC systems are shown in Fig. 1, in which the OOK modulation is employed in both cases due to its simplicity. In these systems, digital signals are firstly coded by RC or STBC encoders, and the resulting signals are used to modulate the optical carriers and sent to two transmitters (known as $\mathrm{Tx}_{1}$ and $\mathrm{Tx}_{2}$ ). The transmitters launch modulated optical signals to the free space, and after free space propagation, the optical signals are detected by a photodetector (PD). The converted electrical signal is then decoded accordingly in offline signal processing.

In this case, the channel gain, $\mathrm{h}_{i}$, from $\operatorname{Tx}_{i}(i=1$ or 2$)$ to $\mathrm{Rx}$, explicitly comprises the electrical to optical modulation gain $\mathrm{h}_{i}^{e-o}$, the optical channel gain $\mathrm{h}_{i}^{o}$ (including the optical fiber link gain $\mathrm{h}_{i}^{o_{-} f}$ and the OWC link gain $\mathrm{h}_{i}^{o_{-}{ }_{-} \text {sum }}$ ) as well as the optical detection gain $\mathrm{h}_{i}^{o-e}$. It is worth noting that, in the optical beam blockage scenario, $\mathrm{h}_{i}^{o_{-} w_{-} \text {sum }}$ comprises two parts: one is the received optical beam aperture gain $\mathrm{h}_{i}^{o_{-} w_{-} a p}$, which captures the effects of beam divergence angle at transmitter, the relative locations of transmitter and receiver, and the FOV of receiver 


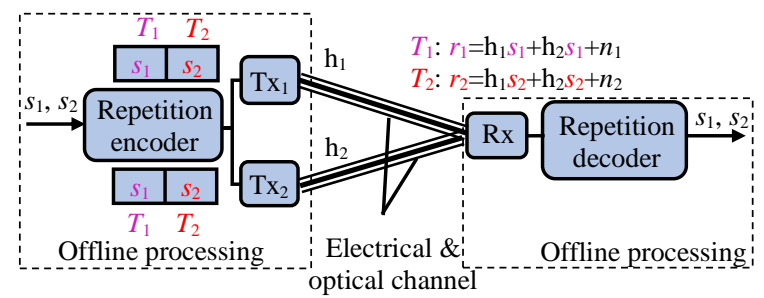

(a)

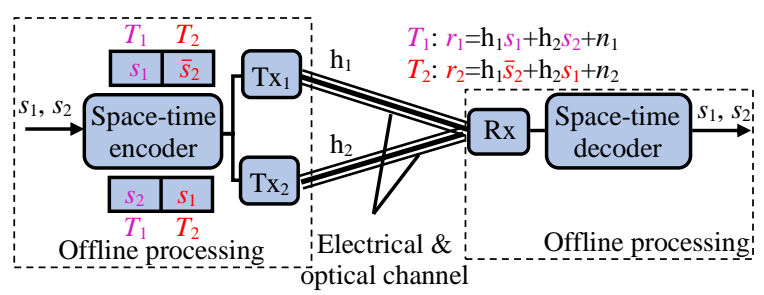

(b)

Tx - Electrical transmitter port; Rx - Electrical receiver port; $s_{1}, s_{2}$ - Transmitted signal; $\bar{s}_{2}$ - Bitwise not of $s_{2} ; r_{1}, r_{2}-$ Received signal; $\mathrm{h}_{1}, \mathrm{~h}_{2}$ - Channel gain; $n_{1}, n_{2}$ - Noise

Fig. 1. The schematic block diagrams of RC and STBC schemes for $2 \times 1$ MISO indoor OWC system: (a) RC and (b) STBC.

etc. [16]. This component is assumed to be a fixed constant at a certain location. The other part of $\mathrm{h}_{i}^{o_{-} w_{-} \text {sum }}$ is the optical wireless channel gain $\mathrm{h}_{i}^{o_{-} w_{-} c}$ induced by the optical power degradation due to the optical beam blockage, which has a significant impact on the system performance. To further simplify the analysis, we assume the external modulation bias is optimized and fixed at the quadrature point and the fiber link length is also fixed. Therefore, $\mathrm{h}_{i}^{o_{-} w_{-} a p}, \mathrm{~h}_{i}^{e-o}, \mathrm{~h}_{i}^{o_{-} f}$ and $\mathrm{h}_{i}^{o-e}$ are constants, and their product is denoted as $\alpha_{i}$. Hence, the total channel gain $\mathrm{h}_{i}$ is given by

$$
\mathrm{h}_{i}=\mathrm{h}_{i}^{e-o} \times \mathrm{h}_{i}^{o_{-} f} \times \mathrm{h}_{i}^{o_{-} w_{-} a p} \times \mathrm{h}_{i}^{o_{-} w_{-} c} \times \mathrm{h}_{i}^{o-e}=\alpha_{i} \mathrm{~h}_{i}^{o-w_{-} c}
$$

For the RC scheme, identical signals are sent from both transmitters in each bit interval, as shown in Fig. 1(a). If the two optical channels are well synchronized, the two optical wireless transmission power levels $\left\langle P_{i}^{t x}\right\rangle(i=1$ or 2$)$ are linearly combined at the PD. Therefore, the photocurrent $I^{r x}$ is illustrated as

$$
I^{r x}=R_{d} \times \sum_{1}^{2} \mathrm{~h}_{i}^{o_{-} w_{-} c}\left\langle P_{i}^{t x}\right\rangle+n
$$

where $R_{d}$ is the photodetector responsivity, $n$ is modeled as the additive white Gaussian noise (AWGN) with main contributions from shot and thermal noises. Therefore, the electrical signal-to-noise ratio (SNR) [6] at the receiver for the $\mathrm{RC}$ scheme is

$$
\begin{aligned}
& \operatorname{SNR}=\frac{\left(R_{d} \times \sum_{1}^{2} \mathrm{~h}_{i}^{o_{-} w_{-} c}\left\langle P_{i}^{t x}\right\rangle\right)^{2}}{\sigma_{\text {shot }}^{2}+\sigma_{\text {thermal }}^{2}} \\
& \cong \frac{\left(R_{d} \times \sum_{1}^{2} \mathrm{~h}_{i}^{o_{-} w_{-} c}\left\langle P_{i}^{t x}\right\rangle\right)^{2}}{2 q R_{d} \times\left(\sum_{1}^{2} \mathrm{~h}_{i}^{o_{-} w_{-} c}\left\langle P_{i}^{t x}\right\rangle+P_{b d}\right) I_{2} \Delta f+\frac{4 k_{B} T}{R_{L}} I_{2} \Delta f}
\end{aligned}
$$

where $\sigma_{\text {shot }}^{2}$ and $\sigma_{\text {thermal }}^{2}$ are the shot and thermal noise variances, respectively, $q$ is the electron charge, $P_{b d}$ is the average background light power, $I_{2}$ is the noise bandwidth factor, $\Delta f$ is the receiver bandwidth, $k_{B}$ is the Boltzmann's constant, $T$ is the absolute temperature, and $R_{L}$ is the equivalent input resistance. For the SNR approximation, we neglect the field-effect transistor (FET) $1 / f$ noise and the FET white channel noise in the thermal noise variance $[6,32]$.

If the two optical wireless channel gains $\mathrm{h}_{i}^{o_{-} w_{-} c}(i=1$ and 2$)$ are known at the receiver, the conditional BER for RC using OOK modulation follows the relationship $[6,17]$

$$
\left.\mathrm{BER}\right|_{\mathrm{h}_{1}^{o} w_{-} c} \text { and } \mathrm{h}_{2}^{o-} w_{-} c=Q(\sqrt{S N R})
$$

where $Q(x)=\frac{1}{\sqrt{2 \pi}} \int_{x}^{\infty} e^{-\frac{y^{2}}{2}} \mathrm{~d} y$.

On the other hand, for the STBC scheme, as shown in Fig. 1(b), during the first bit interval $T_{1}, s_{1}$ is sent from $\operatorname{Tx}_{1}$ and $s_{2}$ is from $\mathrm{Tx}_{2}$, and in the second bit interval $T_{2}, \bar{s}_{2}$ is from $\mathrm{Tx}_{1}$ and $s_{1}$ is from $\mathrm{Tx}_{2}$ [33]. Here, the bitwise-not signal $\bar{s}_{2}$ is used to ensure the non-negative real-value Alamouti-type STBC signal detection. Similarly, in synchronized channels, the two transmitted optical wireless channel powers are linearly combined upon reception. However, since two bit intervals are used for each pair of STBC signals, the photocurrent vector in the two consecutive bit intervals is shown as

$$
\vec{I}^{r x}=R_{d} \times\left(\begin{array}{l}
\sum_{1}^{2} \mathrm{~h}_{i}^{o_{-} w_{-} c}\left\langle P_{i_{-} T_{1}}^{t x}\right\rangle \\
\sum_{1}^{2} \mathrm{~h}_{i}^{o_{-} w_{-} c}\left\langle P_{i_{-} T_{2}}^{t x}\right\rangle
\end{array}\right)+\vec{n}
$$

where the transmission power matrix $\left(\begin{array}{ll}\left\langle P_{1-T_{1}}^{t x}\right\rangle & \left\langle P_{2}^{t x} T_{1}\right\rangle \\ \left\langle P_{1-T_{2}}^{t x}\right\rangle & \left\langle P_{2_{-} T_{2}}^{t x}\right\rangle\end{array}\right)$ corresponds to the modulated electrical signal matrix $\left(\begin{array}{ll}S_{1} & S_{2} \\ s_{2} & s_{1}\end{array}\right)$, and $\vec{n}$ is the noise vector. Using the maximum-likelihood (ML) decision, the electrical SNR at the receiver for the STBC scheme is given by

$$
\begin{aligned}
& \operatorname{SNR}=\frac{\left(R_{d} \times \sqrt{\sum_{1}^{2}\left(\mathrm{~h}_{i}^{o_{-} w_{-} c}\left\langle P_{i}^{t x}\right\rangle\right)^{2}}\right)^{2}}{\sigma_{\text {shot }}^{2}+\sigma_{\text {thermal }}^{2}} \\
& \cong \frac{\left(R_{d} \times \sqrt{\sum_{1}^{2}\left(\mathrm{~h}_{i}^{o_{-} w_{-} c}\left\langle P_{i}^{t x}\right\rangle\right)^{2}}\right)^{2}}{2 q R_{d} \times\left(\sqrt{\sum_{1}^{2}\left(\mathrm{~h}_{i}^{o_{-} w_{-} c}\left\langle P_{i}^{t x}\right\rangle\right)^{2}}+P_{b d}\right) I_{2} \Delta f+\frac{4 k_{B} T}{R_{L}} I_{2} \Delta f}
\end{aligned}
$$

where $\left\langle P_{i}^{t x}\right\rangle=\left\langle P_{i_{-} T_{1}}^{t x}\right\rangle=\left\langle P_{i_{-} T_{2}}^{t x}\right\rangle(i=1$ or 2$)$ is the average transmission power in the corresponding channel. Similarly, the conditional BER for STBC using OOK modulation can also be calculated by (4) [17].

Using the theoretical framework shown by (2)-(4) and (4)(6), the BER performance of both RC and STBC schemes in infrared indoor OWC systems is numerically simulated, and simulation results are shown in Fig. 2. Here, the received optical power is $\sum_{1}^{2} \mathrm{~h}_{i}^{o_{-} w_{-} c}\left\langle P_{i}^{t x}\right\rangle$, which is the total received power from both transmitters. For simplicity, we assume that the two OWC channels have identical transmission optical power levels, expressed as $\mathrm{h}_{1}^{o_{-} w_{-} c}\left\langle P_{1}^{t x}\right\rangle=\mathrm{h}_{2}^{o_{-} w_{-} c}\left\langle P_{2}^{t x}\right\rangle$. When an optical filterbased photodetector is utilized with the responsivity $R_{d}=$ $0.8 \mathrm{~A} / \mathrm{W}$, we assume that the infrared background light power $P_{b d}$ is negligible. The transmission data rate is assumed to be $10 \mathrm{~Gb} / \mathrm{s}$ with $\mathrm{OOK}$ format. It can be seen from the simulation 
results that the RC scheme outperforms the STBC scheme by $\sim 2 \mathrm{~dB}$ in the receiver sensitivity. This is consistent with the theoretical analysis shown in (3) and (6), where $\sum_{1}^{2} \mathrm{~h}_{i}^{o_{-} w_{-} c}\left\langle P_{i}^{t x}\right\rangle>\sqrt{\sum_{1}^{2}\left(\mathrm{~h}_{i}^{o_{-} w_{-} c}\left\langle P_{i}^{t x}\right\rangle\right)^{2}}$ leads to a better SNR in the RC scheme.

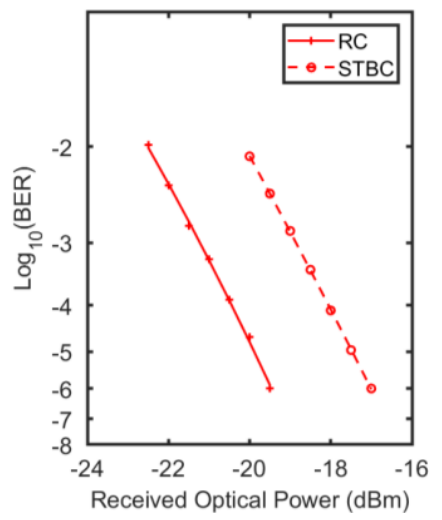

Fig. 2. Simulation results for the RC and STBC schemes with equal optical power distribution in two identical OWC channels.

In the following experimental demonstrations, the measured BER results obtained from different transmitted power distributions using both RC and STBC schemes will further verify the aforementioned theory.

\section{EXPERIMENTS AND RESULTS}

Fig. 3 shows the proof-of-concept experimental setup for verifying the theoretical framework on RC and STBC schemes in infrared indoor OWC systems. RC or STBC encoded OOK signals were generated with an arbitrary waveform generator (AWG, using two separate channels). Then the two $10 \mathrm{~Gb} / \mathrm{s}$ signals were amplified and used to modulated two laser sources with wavelengths of $1550 \mathrm{~nm}$ and $1549 \mathrm{~nm}$, respectively. Two wavelengths were used to avoid the possible interference at the receiver if the same wavelength was used for both channels. This can enable better comparative investigation of the two coding schemes. An optical attenuator was employed after each modulator to vary the transmitted optical power and hence, to emulate power fluctuations due to the optical beam blockage in OWC channels. An optical delay line was also inserted in one of the links to introduce controllable optical path delay. Here, we focused on less than one-bit interval delay caused by channel path difference, since the delay more than one-bit interval can be recovered by channel training, whilst less than 1-bit delay is difficult to deal with since it requires precise synchronization. At each pigtailed fiber-based transmitter end, the optical signal emitting to free space had a $16^{\circ}$ beam divergence with a maximum output power of $4 \mathrm{dBm}$, and hence, the maximum power level of the combined two beams was still under the eye laser safety level [9]. Two OWC beams were close to each other in 1-meter OWC link to ensure both beams were efficiently focused by a specially designed lenses system and then detected by a multimode fiber (MMF) coupled PD. AR-coated lens was used to ensure the visible background light were substantially attenuated. Furthermore, the diffused indoor infrared and visible background light was difficult to be coupled into the MMF coupled PD due to the numerical aperture limit of fiber. Finally, the converted electrical signal was sampled by the digital signal oscilloscope (DSO) for offline signal processing.

The measured BER results with only one active channel are shown in Fig. 4 (a) as a benchmark. This measurement was taken without any optical beam blockage in the OWC channels. Hence, the two optical wireless channel gains were similar, i.e. $\mathrm{h}_{1}^{o_{-} w_{-} c} \cong \mathrm{h}_{2}^{o_{-} w_{-} c}$. From the BER results in Fig. 4 (a), it is obvious that the two total channel gains are similar, i.e. $h_{1} \cong h_{2}$. Therefore, it is deduced from (1) that $\alpha_{1} \cong \alpha_{2}$, and hence, $\mathrm{h}_{1}^{e-o} \times \mathrm{h}_{1}^{o_{-} f} \times \mathrm{h}_{1}^{o_{-} w_{-} a p} \times \mathrm{h}_{1}^{o-e} \cong \mathrm{h}_{2}^{e-o} \times \mathrm{h}_{2}^{o_{-} f} \times \mathrm{h}_{2}^{o_{-} w_{-} a p} \times$

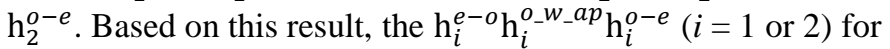
both channels can be considered as a constant, which is used in the following analysis.

With optical beam blockage, the optical wireless channel gains are different. It is worth noting that in our experiment, the optical power in the fiber link was attenuated to emulate the optical power degradation induced by the optical beam blockage, and hence, the different optical wireless channel gains (i.e. $\mathrm{h}_{1}^{o_{1} w_{-} c} \neq \mathrm{h}_{2}^{o_{-} w_{-} c}$ ) in the previous analysis are equivalent to the different fiber link gains (i.e. $\mathrm{h}_{1}^{o_{-} f} \neq \mathrm{h}_{2}^{o_{-} f}$ ). The reason for this equivalent analysis is that the received optical power variation is caused by varying the transmission optical power allocations, and this received power can be rewritten from (1) as

$$
\sum_{1}^{2} \mathrm{~h}_{i}^{o_{-} w_{-} c}\left\langle P_{i}^{t x}\right\rangle=\frac{1}{\mathrm{~h}^{e-o} \mathrm{~h}_{-}^{o_{-} w_{-} a p} \mathrm{~h}^{o-e}} \sum_{1}^{2} \frac{\left\langle P_{i}^{t x}\right\rangle}{\mathrm{h}_{i}^{o-f}} \mathrm{~h}_{i}
$$

where $\mathrm{h}^{e^{-o}} \mathrm{~h}^{o_{-} w_{-} a p} \mathrm{~h}^{o-e}$ is a constant in two channels according to the above analysis. From (7), varying the optical wireless channel gains $\mathrm{h}_{i}^{o_{-} w_{-} c}$ in the experiment can be achieved by varying the fiber link gain $\mathrm{h}_{i}^{o_{-} f}$, and the term $\frac{\left\langle P_{i}^{t x}\right\rangle}{\mathrm{h}_{i}^{o-f}}$ is the fixed optical power from the laser source.

To investigate the performance of the RC and STBC schemes in different optical beam blockage scenarios, two types of transmission power distributions are firstly studied in the two channels, i.e. with equal power distribution and with $3 \mathrm{~dB}$ difference in power distribution. Here, we focus on the impact of beam blockage, therefore, the two channel links are synchronized, i.e., no optical path delay. The channel gains $\mathrm{h}_{i}$ were estimated via averaging the values of non-overlapped training sequences appended to the corresponding data frames for the two channels at the receiver. The BER results for the RC scheme are shown by the two blue curves in Fig. 4 (b). It is clear that for the RC scheme, as long as the total transmitted optical powers are kept constant $\left(\sum_{1}^{2} \mathrm{~h}_{i}^{o_{-} w_{-} c}\left\langle P_{i}^{t x}\right\rangle=\right.$ constant $)$, the same BER can be maintained regardless of the power distributions. This is consistent with the theory shown in Section II, since as shown by (3), the same $\sum_{1}^{2} \mathrm{~h}_{i}^{o_{-}{ }_{-} c}\left\langle P_{i}^{t x}\right\rangle$ results in the same SNR. Therefore, it can be implied that the RC scheme can alleviate optical power degradation caused by partial beam blockage via providing extra powers from another transmitter. Besides, the BER for STBC with $3 \mathrm{~dB}$ difference power distribution is 


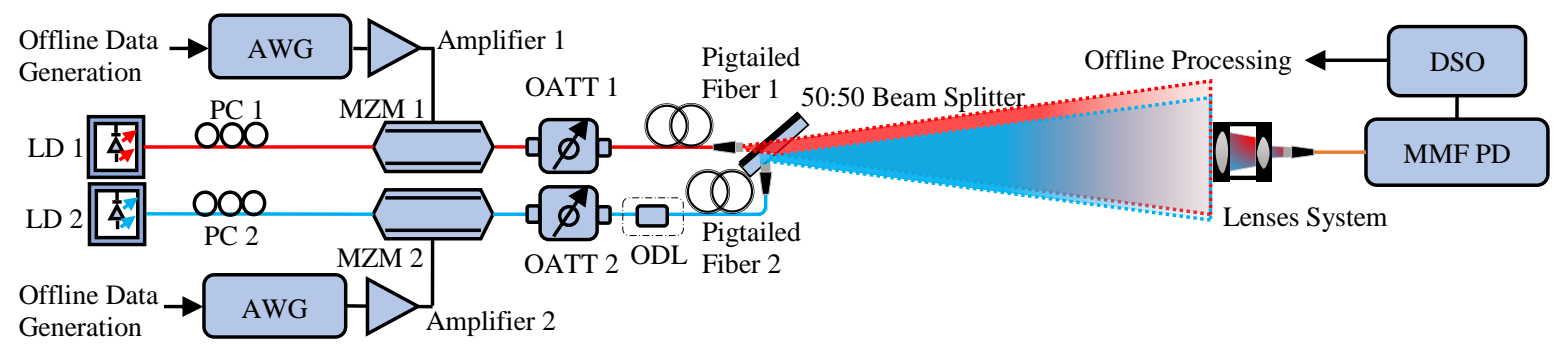

AWG - Arbitrary Waveform Generator; LD - 1550nm infrared laser; PC - Polarization Controller; MZM - Mach Zehnder Modulator; OATT - Optical Attenuator;ODL - Optical Delay Line; MMF - Multimode Fiber; PD - Photodetector; DSO - Digital Storage Oscilloscope

Fig. 3. Experimental setup for demonstrating the RC and STBC schemes.

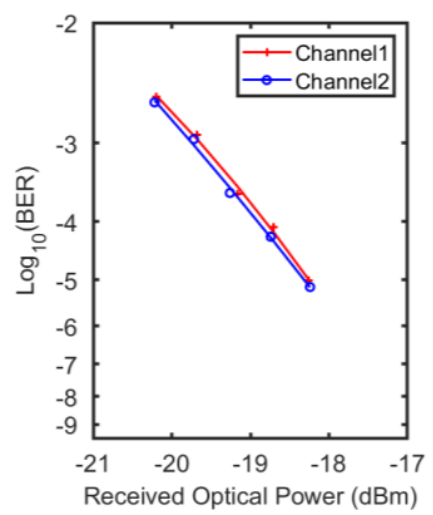

(a)

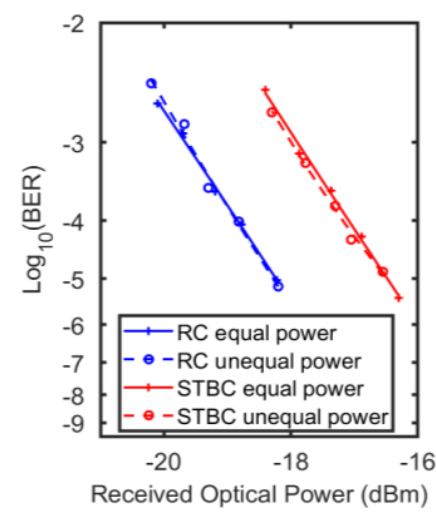

(b)
Fig. 4. (a) BER for only one active channel. (b) BER for the RC and STBC schemes with equal power distribution and with $3 \mathrm{~dB}$ difference power distribution (two channels are synchronized).

theoretically better than STBC with equal power distribution even if the total received power $\sum_{1}^{2} \mathrm{~h}_{i}^{o_{-} w_{-} c}\left\langle P_{i}^{t x}\right\rangle$ remains constant. This is due to the dependence of the SNR on

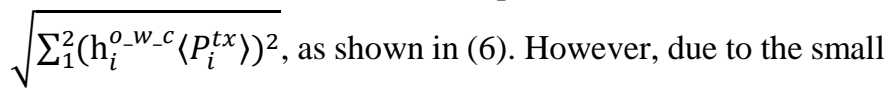
received optical power in the STBC experiment, the BER difference is difficult to observe. Therefore, it is shown in Fig. 4 (b) for the STBC scheme (two red curves), the tolerance to $3 \mathrm{~dB}$ difference unequal power distributions is similar to that of RC (two blue curves). In such case, more detailed investigation on the BER performance of different transmitted power distributions using STBC scheme will be studied in the following paragraph. In addition, it is clear from Fig. 4 (b) that comparing the two transmitter diversity schemes, the system with RC outperforms the STBC system by $\sim 2 \mathrm{~dB}$ in the receiver sensitivity regardless of the power distributions, and this agrees with the simulation results shown in Fig. 2. Furthermore, it is obvious from Fig. 4 (b) that without optical beam blockage, both schemes are capable of extending the optical beam coverage at the receiver plane by steering the two optical beams [12-13] to different areas and adjusting the transmitted optical power levels. In this case, the receiver at different locations can either receive both transmitted signals in the beam overlapping area for diversity, or receive only one of transmitted signals in beam non-overlapping area for multiplexing [14]. It is worth noting that it is not always necessary for one receiver to be only served by one transmitter at a time, since fully link blockage is not always encountered. Beam overlapping with spatial diversity can be an alternative, which reduces the operation of beam steering, leading to reduced system coordination complexity as well as be more tolerant to localization accuracy.

To find the BER trend for the STBC scheme under constant total received power, more cases of power distributions between the two synchronized channels were investigated, and the measured BER are shown in Fig. 5 (a). Here, in addition to the $3 \mathrm{~dB}$ power difference between the two channels as considered in Fig. 4 (b), two more cases were studied, which are: 1. One transmitted power is fixed at a low level ($23.07 \mathrm{dBm}$ ), and the other transmitted power is increased gradually from $-20.07 \mathrm{dBm}$ to $-17.64 \mathrm{dBm} ; 2$. One transmitted power is fixed at a high level $(-18.84 \mathrm{dBm})$, and the other transmitted power is decreased gradually from $-20.29 \mathrm{dBm}$ to $28.20 \mathrm{dBm}$. It can be seen that the worst BER is achieved with equal power distribution. The reason is discussed as follows.

Fig. 5 (b) shows the sum of the measured total channel gains $\sum_{1}^{2} \mathrm{~h}_{i}$ with different received optical powers $\sum_{1}^{2} \mathrm{~h}_{i}^{o-w_{-} c}\left\langle P_{i}^{t x}\right\rangle$. It can be seen that regardless of the power distribution, $\sum_{1}^{2} \mathrm{~h}_{i}$ is always proportional to $\sum_{1}^{2} \mathrm{~h}_{i}^{o_{-} w_{-} c}\left\langle P_{i}^{t x}\right\rangle$, and is given by

$$
\sum_{1}^{2} \mathrm{~h}_{i}^{o-w_{-} c}\left\langle P_{i}^{t x}\right\rangle \propto \sum_{1}^{2} \mathrm{~h}_{i}
$$

which agrees with the equivalent model proposed in (7). Besides, it is clear that in the experiment, $\mathrm{Q}\left(\sqrt{\sum \mathrm{h}_{i}^{2}}\right)$ has an approximate linear relationship with the $\log _{10}$ (BER), that is

$$
\mathrm{Q}\left(\sqrt{\sum h_{i}^{2}}\right) \propto \log _{10}(\mathrm{BER})
$$

The results as shown in Fig. 5(c) and (9) match the theoretical analysis given in (4) and (6), which show that a small variation of $\sqrt{\sum \mathrm{h}_{i}^{2}}$ leads to an approximately linear relationship with $\log _{10}$ (BER). Moreover, based on the investigation of the measured channel gains as shown in Fig. 5 (b)-(c), the relationship between $\sum_{1}^{2} \mathrm{~h}_{i}$ and $\mathrm{Q}\left(\sqrt{\sum \mathrm{h}_{i}^{2}}\right)$ is depicted in Fig. 5 (d), which roughly follows the BER trend in Fig. 5 (a), thus validating the above analysis. In addition, from Fig. 5 (a), it is indicated that the equal transmitted optical power distribution in the STBC scheme results in the worst BER performance. That is because that equal transmitted power distribution leads to identical channel gains $h_{1}=h_{2}$, and hence from Fig. $5(d)$, the value of $\mathrm{Q}\left(\sqrt{\sum \mathrm{h}_{i}^{2}}\right)$ is the maximum (i.e., minimum $\left.\sqrt{\sum \mathrm{h}_{i}^{2}}\right)$, which leads to the worst BER performance. 


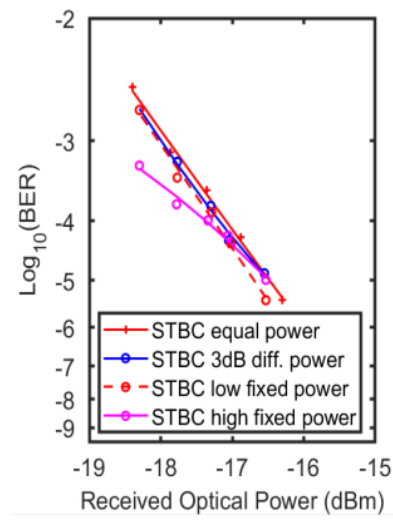

(a)

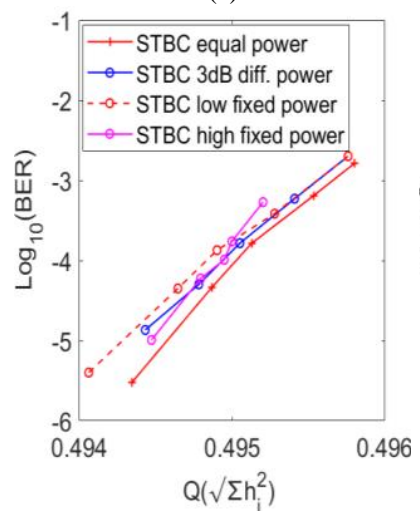

(c)

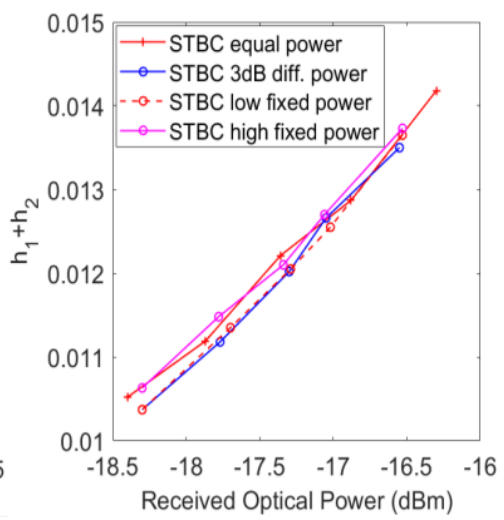

(b)

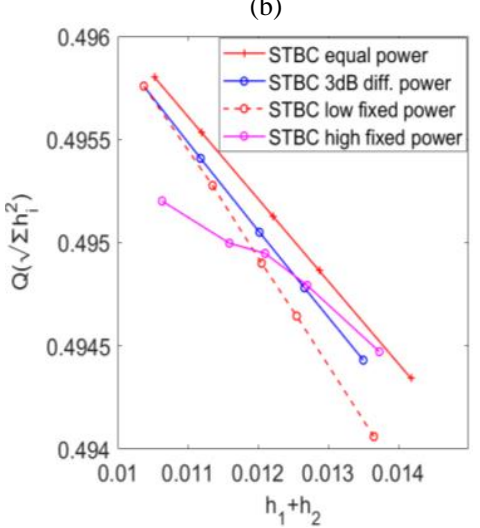

(d)
Fig. 5. (a) BER for the STBC scheme with different power distributions between the two synchronized channels. (b) Relationship between the received optical power and the sum of channel gains $\sum_{1}^{2} h_{i}$. (c) Relationship between $\mathrm{Q}\left(\sqrt{\sum \mathrm{h}_{i}^{2}}\right)$ and BER. (d) Relationship between $\sum_{1}^{2} \mathrm{~h}_{i}$ and $\mathrm{Q}\left(\sqrt{\sum \mathrm{h}_{i}^{2}}\right)$.

Due to user mobility, it is very likely that the two optical channels have different path lengths, which results in the optical delay in received signals and imperfect synthronisation. As discussed above, less than one-bit interval optical delay is investigated in this paper on the RC and STBC schemes since more than one-bit interval delay can be tackled by channel training. Here, this optical delay within one-bit interval was emulated by changing the controllable optical delay line at one of the transimitters. The transmitted data rate was kept at 10 $\mathrm{Gb} / \mathrm{s}$ with OOK modulation, and the sampling rate of the DSO at the receiver was $40 \mathrm{GS} / \mathrm{s}$ with oversampling factor=4. The measured BER performance is shown in Fig. 6, where $x / 5$ ( $x=1,2$ or 3 ) optical delay was measured to better illustrate the BER trend in the neighborhood of $1 / 2$ bit optical delay. It can be seen from Fig. 6 that within 3/5 bit optical delay, both RC and STBC schemes can keep an adequate communication link performance, with a faster BER deterioration speed at higher optical delays. This is because for the OOK modulation without extra guard intervals or pulse shaping, the two transmission signals will always overlap in the DSO (oversampling factor=4) within $3 / 5$ bit delay. Fig. 7 shows the schematic diagram of how the DSO oversamples each bit. Note that noise is not considered here for better illustration. It can be seen that for each four oversampling values in one-bit interval, the second and third values provide better approximation to the true value of the bit, as shown in the red dash box in Fig. 7. When the optical delay is increased, the sampled received signals are subjected to larger deviation from the true value of the synchronized signals, resulting in a faster BER deterioration, especially when the delay is larger than $1 / 2$ bit (the second and third oversampled value of the two transmitted signals are not overlapped). Besides, it is worth noting that, for the STBC scheme, when the delay between two channels increases, the BER performance degradation is slightly faster compared to that of RC. This is due to the coding and decoding principles of the real-valued Alamouti-type STBC, three signal levels exist upon detection decision rather than two signal levels in the RC scheme, as illustrated in Fig. 8. Therefore, the probability of decision error is larger in the STBC scheme for delayed signals.

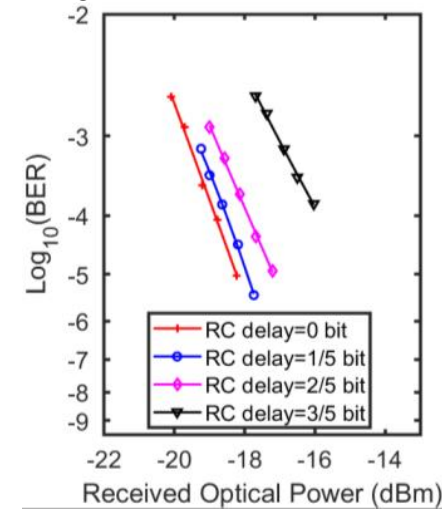

(a)

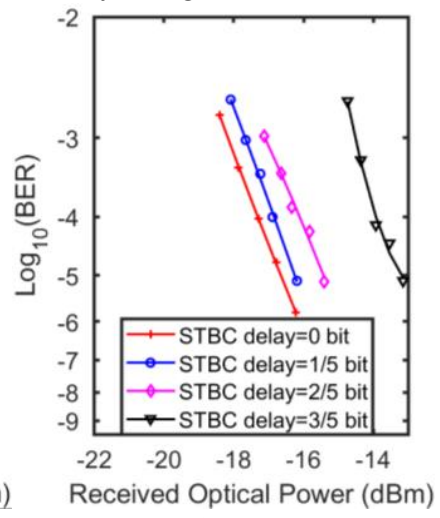

(b)
Fig. 6. Optical delay within one-bit interval using OOK modulation. (a) RC scheme. (b) STBC scheme.

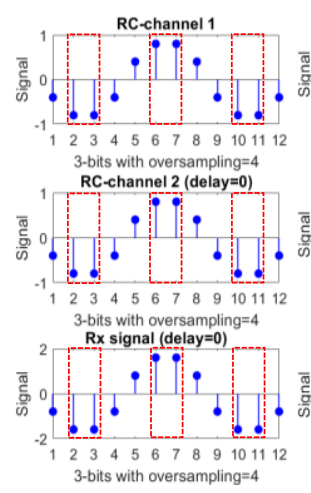

(a)

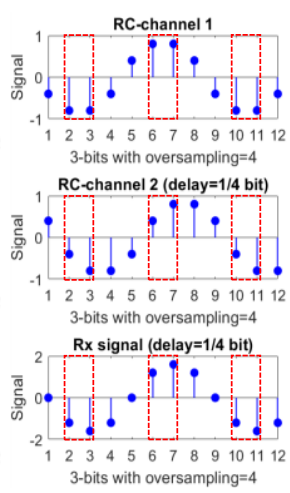

(b)

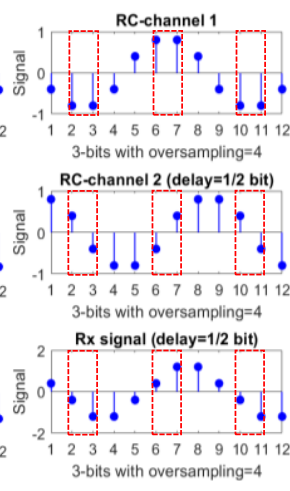

(c)
Fig. 7. Illustration of the sampling process at the receiver in the $\mathrm{RC}$ scheme example with and without delay (oversampling factor=4 in the oscilloscope). (a) Delay $=0$. (b) Delay=1/4 bit. (c) Delay $=1 / 2$ bit.

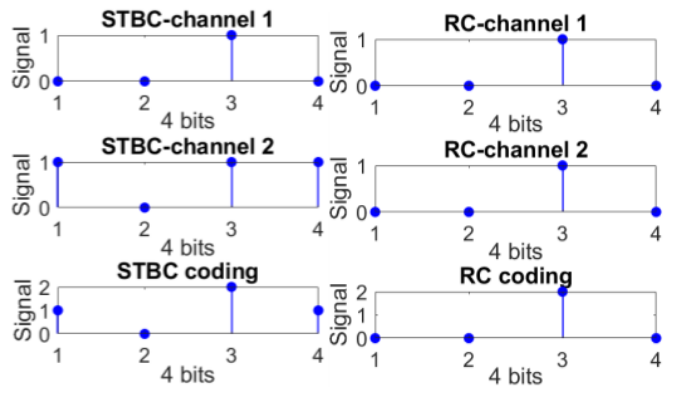

(a)

(b)

Fig. 8. Signal level. (a) STBC coding. (b) RC coding. 
Based on the above analysis, it is clear that with a fixed total received optical power, the RC scheme has the same BER performance regardless of the power distribution between two channels, and it outperforms the STBC scheme where the BER performance varies with the power distribution. The experimental results agree well with the theoretical framework developed in Section II. Besides, when the optical delay within one-bit interval exists, RC also outperforms STBC with better receiver sensitivity and slower BER performance degradation.

\section{CONCLUSION}

We have investigated the RC and STBC schemes in a $2 \times 1$ MISO indoor OWC system as potential transmitter diversity techniques to reduce the impact of optical beam blockage in OWC links. We have evaluated the channel gain components and studied the theoretical model to analyse the relationships between the channel gains, the received optical power and the BER performance. The proposed theoretical models are then validated by proof-of-concept experiments. We have investigated BER results of the RC and STBC schemes under different optical beam blockages emulated by different transmitted power distributions. Furthremore, we have also studied the impact of optical delay within one-bit interval caused by the difference in optical channel path for both RC and STBC schemes. OOK transmission results show that, both RC and STBC schemes exhibit typical gradual drops in BER for delay levels below 3/5-bit interval (oversampling factor=4), and that the linear addition of channel gains in the RC scheme outperforms the root-sum-square of channel gains in the STBC scheme with regards to BER. Future research will focus on the investigation of the delay-tolerant coding schemes to combat optical delay with more than one-bit interval to achieve more robust transmitter diversity, and multiple transmitter configurations to provide large-scale transmitter diversity.

\section{REFERENCES}

[1] M. Z. Chowdhury, M. T. Hossan, A. Islam, and Y. M. Jang, "A comparative survey of optical wireless technologies: architectures and applications," IEEE Access, vol. 6, pp. 9819-9840, 2018.

[2] L. Feng, H. Yang, R. Q. Hu, and J. Wang, "mmWave and VLC-Based Indoor Channel Models in 5G Wireless Networks," IEEE Wireless Communications, no. 99, pp. 2-9, 2018.

[3] O. Bouchet et al., "Optical wireless technology for Terabit's capability with virtual reality use case," in Laser Communication and Propagation through the Atmosphere and Oceans VII, 2018, vol. 10770, p. 1077008: International Society for Optics and Photonics.

[4] K. A. Mekonnen, E. Tangdiongga, and A. Koonen, "High-Capacity Dynamic Indoor All-Optical-Wireless Communication System Backed up With Millimeter-Wave Radio Techniques," Journal of Lightwave Technology, vol. 36, no. 19, pp. 4460-4467, 2018.

[5] A. Nirmalathas, T. Liang, S. Edirisinghe, C. Lim, E. Wong, K. Wang, and K. Alameh, "High-speed optical wireless communications for local area networks (invited)," in Opto-Electronics and Communications Conference (OECC), 2018, 2018.

[6] J. M. Kahn and J. R. Barry, "Wireless infrared communications," Proceedings of the IEEE, vol. 85, no. 2, pp. 265-298, 1997.

[7] H. Elgala, R. Mesleh, and H. Haas, "Indoor optical wireless communication: potential and state-of-the-art," IEEE Communications Magazine, vol. 49, no. 9, 2011.

[8] Z. Ghassemlooy, S. Arnon, M. Uysal, Z. Xu, and J. Cheng, "Emerging optical wireless communications-advances and challenges," IEEE Journal on Selected Areas in Communications, vol. 33, no. 9, pp. 17381749, 2015.
[9] AS/NZS 2211.1:2004, Safety of laser products (Standards Australia International Ltd and Standards New Zealand, 2004).

[10] T. Koonen, "Indoor optical wireless systems: technology, trends, and applications," Journal of Lightwave Technology, vol. 36, no. 8, pp. 14591467,2018

[11] K. Mekonnen, Z. Cao, N. Calabretta, E. Tangdiongga, and A. Koonen, "50 Gb/s indoor optical wireless communication equipped with millimeter-wave backup system and localization and tracking," in 2017 European Conference on Optical Communication (ECOC), 2017, pp. 13: IEEE.

[12] T. Koonen, J. Oh, K. Mekonnen, Z. Cao, and E. Tangdiongga, "Ultra-high capacity indoor optical wireless communication using 2D-steered pencil beams," Journal of Lightwave Technology, vol. 34, no. 20, pp. 4802-4809, 2016.

[13] F. Feng, P. Sangwongngam, H. Chun, G. Faulkner, and D. O'Brien, "Optical Broadcasting for Wide Field-of-View Bidirectional Indoor Optical Wireless Communications," in CLEO: Science and Innovations, 2019, p. SM2G. 5: Optical Society of America.

[14] L. Zheng and D. N. C. Tse, "Diversity and multiplexing: A fundamental tradeoff in multiple-antenna channels," IEEE Transactions on information theory, vol. 49, no. 5, pp. 1073-1096, 2003.

[15] S. M. Navidpour, M. Uysal, and M. Kavehrad, "BER performance of freespace optical transmission with spatial diversity," IEEE transactions on Wireless Communications, vol. 6, no. 8, 2007.

[16] Z. Ghassemlooy, W. Popoola, and S. Rajbhandari, Optical wireless communications: system and channel modelling with Matlab®. CRC press, 2012.

[17] M. Safari and M. Uysal, "Do We Really Need OSTBCs for Free-Space Optical Communication with Direct Detection?" IEEE Transactions on Wireless Communications, vol. 7, no. 11, pp. 4445-4448, 2008.

[18] S. M. Haas, J. H. Shapiro, and V. Tarokh, "Space-time codes for wireless optical communications," EURASIP J. Appl. Signal Process., vol. 3, pp. 1-11, Mar. 2002.

[19] G. Ntogari, T. Kamalakis, and T. Sphicopoulos, "Analysis of indoor multiple-input multiple-output coherent optical wireless systems," Journal of Lightwave technology, vol. 30, no. 3, pp. 317-324, 2012.

[20] R. Priyadarshani, M. Bhatnagar, Z. Ghassemlooy, and S. Zvanovec, "Effect of Correlation on BER Performance of the FSO-MISO System With Repetition Coding Over Gamma-Gamma Turbulence," IEEE Photonics Journal, vol. 9, no. 5, pp. 1-15, 2017.

[21] Y. Ren et al., "Atmospheric turbulence mitigation in an OAM-based MIMO free-space optical link using spatial diversity combined with MIMO equalization," Optics letters, vol. 41, no. 11, pp. 2406-2409, 2016.

[22] T. A. Tsiftsis, H. G. Sandalidis, G. K. Karagiannidis, and M. Uysal, "Optical wireless links with spatial diversity over strong atmospheric turbulence channels," IEEE Transactions on Wireless Communications, vol. 8, no. 2, pp. 951-957, 2009.

[23] A. Street, P. Stavrinou, D. O'brien, and D. Edwards, "Indoor optical wireless systems-a review," Optical and Quantum Electronics, vol. 29, no. 3, pp. 349-378, 1997.

[24] R. J. Green, H. Joshi, M. D. Higgins, and M. S. Leeson, "Recent developments in indoor optical wireless systems," IET communications, vol. 2, no. 1, pp. 3-10, 2008.

[25] C.-C. Wei, F.-M. Wu, Z.-Y. Chen, C.-T. Lin, Y.-S. Huang, and S. Chi, "Indoor VLC System With Multiple LEDs of Different Path Lengths Employing Space-Time Block-Coded DMT/CAP Modulation," Journal of Optical Communications and Networking, vol. 7, no. 3, pp. A459A466, 2015.

[26] L. Qiao, X. Lu, S. Liang, and N. Chi, "MISO visible light communication system utilizing MCMMA aided pre-convergence of STBC decoding," in 2018 Optical Fiber Communications Conference and Exposition (OFC), 2018, pp. 1-3: IEEE.

[27] P. H. Pathak, X. Feng, P. Hu, and P. Mohapatra, "Visible light communication, networking, and sensing: A survey, potential and challenges," IEEE communications surveys \& tutorials, vol. 17, no. 4, pp. 2047-2077.

[28] P. W. Berenguer et al., "Optical Wireless MIMO Experiments in an Industrial Environment," IEEE Journal on Selected Areas in Communications, vol. 36, no. 1, pp. 185-193, 2018.

[29] P. Deng and M. Kavehrad, "Software defined adaptive MIMO visible light communications after an obstruction," in Optical Fiber Communications Conference and Exhibition (OFC), 2017, 2017, pp. 1-3: IEEE. 
[30] F. Zafar, M. Bakaul, and R. Parthiban, "Laser-diode-based visible light communication: Toward gigabit class communication," IEEE Communications Magazine, vol. 55, no. 2, pp. 144-151, 2017.

[31] T. Song, K. Wang, A. Nirmalathas, C. Lim, E. Wong, and K. Alameh, "Demonstration of Indoor Optical Wireless Communications with Spatial Diversity Using Repetition-Coding and Space-Time-Block-Coding," in Opto-Electronics and Communications Conference (OECC), 2018, 2018.

[32] K. Wang, A. Nirmalathas, C. Lim, and E. Skafidas, "Impact of background light induced shot noise in high-speed full-duplex indoor optical wireless communication systems," Optics express, vol. 19, no. 22, pp. 21321-21332, 2011.

[33] M. K. Simon and V. A. Vilnrotter, "Alamouti-type space-time coding for free-space optical communication with direct detection," IEEE Transactions on Wireless Communications, vol. 4, no. 1, pp. 35-39, 2005. 